\title{
Dielectric relaxation and ultrafast transient absorption spectroscopy of $\left[\mathrm{C}_{6} \mathrm{mim}\right]^{+}\left[\mathrm{Tf}_{2} \mathrm{~N}\right]^{-} /$acetonitrile mixtures
}

\author{
Peter W. Lohse, ${ }^{a b}$ Nils Bartels, ${ }^{b}$ Alexander Stoppa,${ }^{c}$ Richard Buchner, ${ }^{* c}$ \\ Thomas Lenzer ${ }^{a}$ and Kawon Oum ${ }^{* a}$
}

Received 23rd November 2011, Accepted 18th January 2012

DOI: $10.1039 / \mathrm{c} 2 \mathrm{cp} 23704 \mathrm{k}$

\begin{abstract}
Mixtures of the ionic liquid (IL) $\left[\mathrm{C}_{6} \mathrm{mim}\right]^{+}\left[\mathrm{Tf}_{2} \mathrm{~N}\right]^{-}$and acetonitrile have been investigated by a combination of dielectric relaxation spectroscopy (DRS) and ultrafast transient absorption techniques using the molecular probe $12^{\prime}$-apo- $\beta$-carotenoic- $12^{\prime}$-acid $\left(12^{\prime} \mathrm{CA}\right)$. Steady-state absorption spectra of the $12^{\prime} \mathrm{CA}$ molecule have also been recorded. The position of the probe's $\mathrm{S}_{0} \rightarrow \mathrm{S}_{2}$ absorption maximum correlates linearly with the polarizability of the mixture, suggesting that the bulk composition is a good approximation to the local composition. The lifetime $\tau_{1}$ of the $\mathrm{S}_{1} / \mathrm{ICT}$ state of $12^{\prime} \mathrm{CA}$ varies rather smoothly with composition between the value for pure acetonitrile (42 ps) and neat $\left[\mathrm{C}_{6} \mathrm{mim}\right]^{+}\left[\mathrm{Tf}_{2} \mathrm{~N}\right]^{-}$(94 ps). At low IL contents there appears to be an influence of discrete ion pairs. Employing static dielectric constants from the DRS experiments, one finds that the lifetime of the probe in the IL mixtures is shorter than that in pure organic solvents with the same polarity parameter. This suggests an increased stabilization of the $\mathrm{S}_{1} / \mathrm{ICT}$ state in IL-containing mixtures, most likely due to IL-specific Coulombic interactions between the cation and the negative end of the probe's dipole. An ultrafast solvation component is observed which is $c a .0 .5 \mathrm{ps}$ in pure acetonitrile, and approaches the value for the pure IL (2.0 ps) already around $x(\mathrm{IL})=0.3$. This is interpreted in terms of an efficient perturbation of the cooperative solvation response of acetonitrile by the presence of small amounts of IL and possibly also the viscosity increase when adding IL. This view is also supported by the increase of the average longitudinal relaxation time of acetonitrile upon addition of small IL amounts extracted from the DRS experiments.
\end{abstract}

\section{Introduction}

Currently, experimental information regarding the polarity and solvation dynamics of ILs and their mixtures with other solvents is still sparse and rather heterogeneous. For instance, extrapolations by dielectric relaxation spectroscopy (DRS) for imidazolium- and pyrrolidinium-based ILs reported by the Weingärtner and Buchner groups provide static dielectric constants between 10 and 18 (comparable to $n$-butanol to $n$-heptanol), with the exception of alkylsulfates and alkylphosphates, which are in the range $30-35 .{ }^{1-3}$ DRS applies an external "macroscopic" alternating field to determine the frequency-dependent complex dielectric function of a sample. Accurate data can be usually obtained in the frequency range

${ }^{a}$ Universität Siegen, Physikalische Chemie, Adolf-Reichwein-Str. 2, 57076 Siegen, Germany.E-mail: oum@chemie.uni-siegen.de; Fax: + 49271740 2803; Tel: + 492717402805

${ }^{b}$ Institut für Physikalische Chemie, Georg-August-Universität Göttingen, 37077 Göttingen, Germany

${ }^{c}$ Institut für Physikalische und Theoretische Chemie, Universität Regensburg, 93040 Regensburg, Germany.

E-mail: Richard.Buchner@chemie.uni-regensburg.de;

Fax: + 49941943 4532; Tel: + 499419434031 from $c a .100 \mathrm{GHz}$ down to $100 \mathrm{MHz}{ }^{4,5}$ Complementary techniques, such as Optical Kerr Effect (OKE) and Terahertz (THz) spectroscopy, can extend this range up to $c a .10 \mathrm{THz} .^{5-7}$ DRS provides not only static dielectric constants, but also, e.g., accurate frequencies of solvent modes, which can be assigned with the aid of sophisticated molecular dynamics simulations. ${ }^{8-10}$

Complementary approaches use local, "microscopic" molecular probes. This concept is more closely linked to chemical reactivity, because it is the local surrounding or the "cybotactic" region of a molecule which crucially influences the outcome of reactions. Probing of the cybotactic region can e.g. be performed by employing solvatochromic absorption probes. ${ }^{11-14}$ Care has to be taken in the interpretation of such steady-state spectroscopic studies when using solvatochromic probes, such as Reichardt's dye $\left(E_{\mathrm{T}}{ }^{\mathrm{N}}\right.$ polarity scale), or the Kamlet-Taft parameter for dipolarity/polarizability, $\pi^{*}$, where the influence of other solvent effects such as hydrogen-bonding may render unambiguous conclusions difficult.

Recently, carbonyl substituted carotenoids were introduced as a new class of dynamic polarity probes, where the lifetime of an excited electronic state smoothly correlates with the dipolarity 


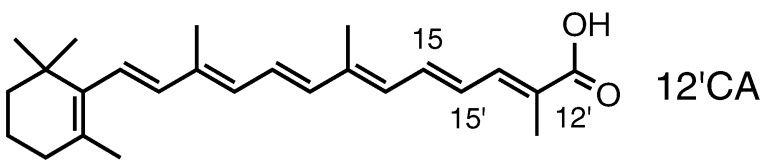

Fig. 1 Chemical structure of $12^{\prime}$-apo- $\beta$-carotenoic- $12^{\prime}$-acid $\left(12^{\prime} \mathrm{CA}\right)$.

of organic solvents, without disturbance by specific solvation effects such as H-bonding. ${ }^{15-19}$ Interestingly, this correlation was not followed by a large range of ILs. ${ }^{20}$ The lifetime of the $\mathrm{S}_{1} / \mathrm{ICT}$ state in these ILs was comparable to that in solvents such as ethanol or methanol, and thus the probe's local environment appears to be considerably more polar than the dielectric constants from DRS suggest. This is in line with earlier results based on solvatochromic probes. ${ }^{11,12}$ The underlying physical reason for the difference between macroscopic and "local" polarities is still unknown and therefore is an interesting question to us.

In addition, it was very recently demonstrated that the same class of molecular probes reports on the timescales of solvation dynamics. ${ }^{21-23}$ As outlined by Ernsting and co-workers, such an ultrafast probe can be regarded as a local THz emitter/detector, which essentially performs dielectric relaxation spectroscopy in the cybotactic region. ${ }^{24}$ Therefore, it is interesting to compare the results of such studies with timescales for solvent movements obtained from "macroscopic" DRS experiments.

The level of complexity is increased when the transition from neat ILs to IL mixtures is made. Buchner and co-workers have recently reported composition-dependent dielectric spectra of binary IL/IL and IL/organic solvent mixtures. ${ }^{3,25-27}$ Mixtures of room temperature ILs and organic solvents allow a systematic variation of thermodynamic, polarity and solvation properties. Another interesting question is then, whether there is an "ideal" mixing behavior in binary mixtures of ILs or IL + organic solvents in the macroscopic and microscopic regimes. "Ideal" behavior has been experimentally observed for some important thermodynamic quantities, such as viscosity and molar volume. ${ }^{26}$ A related issue is the concept of "preferential solvation" in spectroscopic studies of solvatochromic probes. ${ }^{28,29}$

In this study, we use a combined experimental approach using DRS and ultrafast transient absorption spectroscopy to study solvent polarity and solvation in binary mixtures of 1-nhexyl-3-methylimidazolium bis(trifluoromethylsulfonyl)amide $\left(\left[\mathrm{C}_{6} \mathrm{mim}\right]^{+}\left[\mathrm{Tf}_{2} \mathrm{~N}\right]^{-}\right)$with the polar organic solvent acetonitrile. We use the carbonyl carotenoid $12^{\prime}$-apo- $\beta$-carotenoic- $12^{\prime}$-acid $\left(12^{\prime} \mathrm{CA}\right.$, see Fig. 1$)$ as a micropolarity and local $\mathrm{THz}$ probe. The time-resolved experiments are complemented by steadystate absorption measurements.

\section{Experimental}

Dielectric spectra, $\varepsilon^{*}(\nu)=\varepsilon^{\prime}(\nu)-i \varepsilon^{\prime \prime}(\nu)$ (Fig. 2), where $\varepsilon^{\prime}(\nu)$ is the relative permittivity and $\varepsilon^{\prime \prime}(\nu)$ the dielectric loss, were recorded at $25 \pm 0.05{ }^{\circ} \mathrm{C}$ in the frequency range of $0.2 \leq$ $\nu / \mathrm{GHz} \leq 89$. For 0.2 to $20 \mathrm{GHz}$ a Hewlett-Packard model HP $85070 \mathrm{M}$ dielectric probe system consisting of a HP $8720 \mathrm{D}$ vector network analyzer (VNA) and a HP $85070 \mathrm{M}$ dielectric probe kit was used. ${ }^{30}$ Two waveguide interferometers were used at $27 \leq \nu / \mathrm{GHz} \leq 89 .{ }^{31}$ Additionally, a new reflectometer

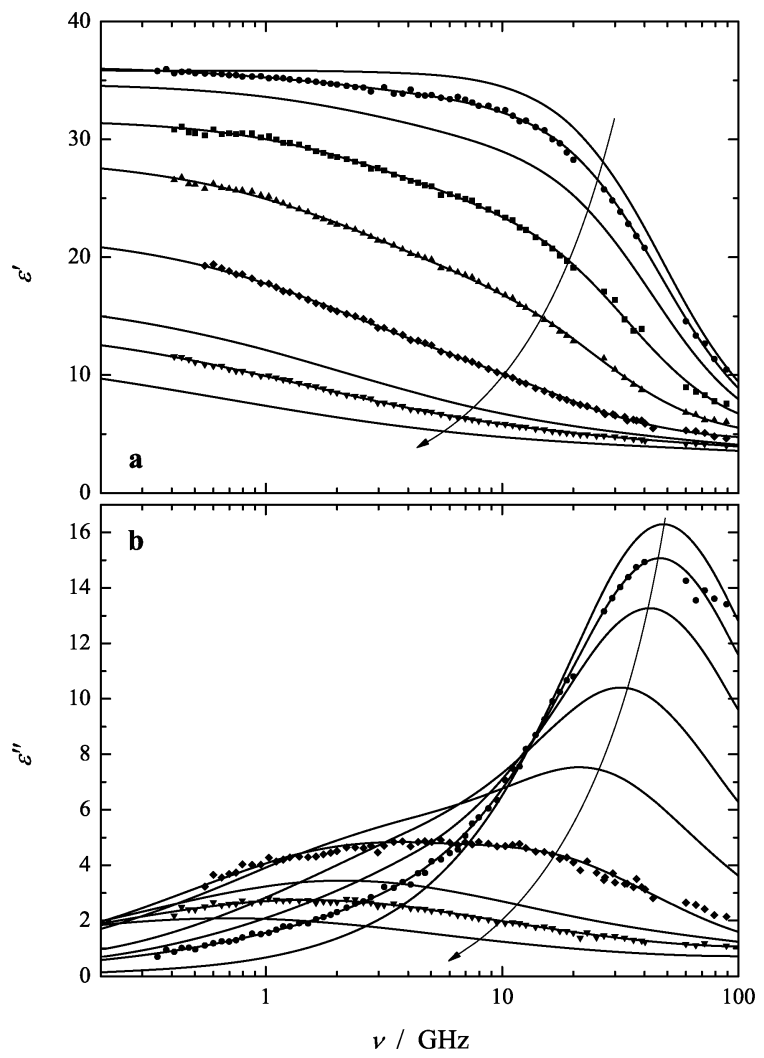

Fig. 2 Spectra of (a) the relative permittivity, $\varepsilon^{\prime}(\nu)$, and (b) the dielectric loss, $\varepsilon^{\prime \prime}(\nu)$, of mixtures of $\left[\mathrm{C}_{6} \mathrm{mim}\right]^{+}\left[\mathrm{Tf}_{2} \mathrm{~N}\right]^{-}+$acetonitrile at $25{ }^{\circ} \mathrm{C}$ and IL mole fractions of $x=0,0.0074,0.0224,0.0577$, $0.1229,0.2595,0.4541,0.5969,1$; increasing in the arrow direction. Lines represent fits with the $\mathrm{CC}+\mathrm{D}$ model. For clarity, experimental data (symbols) are only shown for selected spectra.

consisting of an Agilent E8364B VNA, connected to an electronic calibration module (ECal, Agilent N4693A) and a high-frequency dielectric probe kit $(85070 \mathrm{E})$, was used to cover the region $5 \leq \nu / \mathrm{GHz} \leq 50$ for selected samples. Whilst the interferometers yielded absolute data, the reflection measurements with the VNA probes were calibrated as described in detail previously. ${ }^{2}$ To correct the experimentally accessible total loss, $\eta(\nu)=\varepsilon^{\prime \prime}(\nu)+\kappa /\left(2 \pi \nu \varepsilon_{0}\right)\left(\varepsilon_{0}\right.$ is the electric field constant), of the sample for the contribution from $\mathrm{dc}$ conductivity, $\kappa,{ }^{2}$ the latter was separately determined with the setup described in ref. 32 .

Samples for the DRS studies were prepared from $\left[\mathrm{C}_{6} \mathrm{mim}\right]^{+}\left[\mathrm{Tf}_{2} \mathrm{~N}\right]^{-}$(IoLiTec) dried at a high-vacuum line $\left(p<10^{-8}\right.$ bar) for 7 days at $\sim 40{ }^{\circ} \mathrm{C}$ prior to use (water mass fraction $<50 \mathrm{ppm}$ ) and from acetonitrile (Merck, $>99.9 \%$ ) distilled from $\mathrm{CaH}_{2}$ and stored over activated $4 \AA$ molecular sieves. Solutions were prepared individually on an analytical balance without buoyancy corrections. Solutions were transferred to the DRS apparatus using syringe techniques. Sample preparation, handling and measurement were performed under dry $\mathrm{N}_{2}(\mathrm{~g})$.

Details of the Pump-Supercontinuum Probe (PSCP) setup in Siegen for recording broadband transient absorption spectra can be found in previous publications. ${ }^{21,33-36} 12^{\prime} \mathrm{CA}$ was dissolved in the solvent or solvent mixture of interest and pumped through a thermostatted (298.15 K) quartz flow-through 
cuvette (path length $1.0 \mathrm{~mm}$, window thickness $1.2 \mathrm{~mm}$ ). The reporter molecule was excited at $480 \mathrm{~nm}\left(0.8-3 \mu \mathrm{J}\right.$ pulse $\left.{ }^{-1}\right)$ and the subsequent ultrafast dynamics probed by a supercontinuum $(340-770 \mathrm{~nm})$ at magic-angle polarization. In the current experiments, the cross-correlation time was $c a .80 \mathrm{fs}$ and the time accuracy $10 \mathrm{fs}$. VIS pump (430 nm, <0.1 nJ pulse $\left.{ }^{-1}\right)$-near IR probe $\left(860 \mathrm{~nm},<1 \mathrm{~nJ}\right.$ pulse $\left.^{-1}\right)$ laser experiments were performed with another setup described previously using the same flow-cell. ${ }^{17,37}$ The time resolution of this setup was $c a .130 \mathrm{fs}$. The $12^{\prime} \mathrm{CA}$ probe ( $>97 \%$ all-trans form) was generously provided by Dr Hansgeorg Ernst (BASF SE). $\left[\mathrm{C}_{6} \mathrm{mim}\right]^{+}\left[\mathrm{Tf}_{2} \mathrm{~N}\right]^{-}$ was purchased from IoLiTec and kept over molecular sieves (water mass fraction $<100 \mathrm{ppm}$ ). Acetonitrile (Merck) had a specified purity of $99.5 \%$. All samples were handled under an argon atmosphere. Steady-state absorption spectra were recorded using a Cary $5 \mathrm{E}$ spectrometer.

\section{Results and discussion}

\subsection{Dielectric data}

The dielectric spectra of $\left[\mathrm{C}_{6} \mathrm{mim}\right]^{+}\left[\mathrm{Tf}_{2} \mathrm{~N}\right]^{-}+$acetonitrile mixtures (Fig. 2) change smoothly from the single Debye-type (D) relaxation of pure acetonitrile ${ }^{38}$ centred at $\sim 50 \mathrm{GHz}$ to the broad distribution of relaxation times for the IL. In the investigated frequency range $((0.2$ to 89$) \mathrm{GHz})$ the IL spectrum can be modelled by the superposition of a lowfrequency Cole-Cole (CC) mode at $\sim 0.6 \mathrm{GHz}$ and a small Debye relaxation at $\sim 200 \mathrm{GHz}^{2}$ For imidazolium ILs, including $\left[\mathrm{C}_{6} \mathrm{mim}\right]^{+}\left[\mathrm{Tf}_{2} \mathrm{~N}\right]^{-}$, the low-frequency mode can be essentially assigned to large-angle jump reorientation of the cation. ${ }^{5}$ However, the high-frequency mode is just a formal description of the significant contributions from librations and intermolecular vibrations dominating the far-infrared region. ${ }^{5,8,25}$

Consistent with the bimodal character of the spectra at intermediate concentrations (Fig. 2) and similar to IL + dichloromethane mixtures ${ }^{25}$ all mixture spectra could be well fitted with the $\mathrm{CC}+\mathrm{D}$ model (Fig. 2). We abstain here from giving the parameters (amplitudes, relaxation times and width parameter) of the $\mathrm{CC}+\mathrm{D}$ model as their interpretation for the present IL + acetonitrile mixtures is not straightforward due to strong band overlap. ${ }^{39}$ However, as can be judged from Fig. 2a, the extrapolation $\varepsilon=\lim _{\nu \rightarrow 0} \varepsilon^{\prime}(\nu)$ yielding the static permittivity, $\varepsilon$, of the $\left[\mathrm{C}_{6} \mathrm{mim}\right]^{+}\left[\mathrm{Tf}_{2} \mathrm{~N}\right]^{-}+$ acetonitrile mixtures, is always unproblematic. This key quantity of the present dielectric measurements is given in Table 1, together with the average relaxation time of the samples, $\tau_{\mathrm{av}}=$ $\left(2 \pi \nu_{\text {peak }}\right)^{-1}$, associated with the frequency $\nu_{\text {peak }}$ of their loss peak, $\operatorname{Max}\left[\varepsilon^{\prime \prime}(\nu)\right]$. The significant increase of $\tau_{\mathrm{av}}$ from 5.8 ps at $x=$ 0.08403 to 42 ps at $x=0.2595$ reflects the switch-over from the acetonitrile-dominated spectrum at a low $\left[\mathrm{C}_{6} \mathrm{mim}\right]^{+}\left[\mathrm{Tf}_{2} \mathrm{~N}\right]^{-}$ content to IL-dominated spectra at $x>0.25$.

Fig. 3 shows the dependence of the static permittivity on the IL mole fraction, $x$. As expected from the significant difference in the static permittivities of both compounds $(\varepsilon=35.96$ for pure acetonitrile ${ }^{40}$ and 12.7 for $\left.\left[\mathrm{C}_{6} \mathrm{mim}\right]^{+}\left[\mathrm{Tf}_{2} \mathrm{~N}\right]^{-2}\right), \varepsilon$ increases strongly with decreasing $x$. Interestingly, however, for vanishing IL content $\varepsilon$ does not smoothly extrapolate to the value of pure acetonitrile $(\sim 35.9),{ }^{38,40,41}$ but reaches $\varepsilon=37.0$ at the lowest
Table 1 Static permittivity, $\varepsilon$, and average relaxation time, $\tau_{\mathrm{av}}=$ $\left(2 \pi \nu_{\text {peak }}\right)^{-1}$, of $\left[\mathrm{C}_{6} \mathrm{mim}\right]^{+}\left[\mathrm{Tf}_{2} \mathrm{~N}\right]^{-}+$acetonitrile mixtures as a function of IL mole fraction, $x(\mathrm{IL})$

\begin{tabular}{llr}
\hline$x(\mathrm{IL})$ & $\varepsilon$ & $\tau_{\mathrm{av}} / \mathrm{ps}$ \\
\hline 0 & $35.84^{a}, 35.92^{b}, 35.96^{c}$ & 3.3 \\
0.00235 & 37.0 & 3.4 \\
0.00481 & 36.6 & 3.4 \\
0.00739 & 36.3 & 3.4 \\
0.01009 & 36.2 & 3.5 \\
0.01592 & 35.2 & 3.6 \\
0.02244 & 34.8 & 3.8 \\
0.03795 & 33.0 & 4.3 \\
0.05770 & 31.8 & 5.0 \\
0.08403 & 30.5 & 5.8 \\
0.1229 & 28.6 & 7.4 \\
0.1776 & 26.4 & 10 \\
0.2595 & 22.2 & 42 \\
0.3417 & 20.1 & 69 \\
0.4541 & 17.0 & 76 \\
0.5969 & 14.8 & 106 \\
0.8171 & 13.1 & 207 \\
1 & $12.7^{d}$ & 243 \\
${ }^{a}$ Ref. $41 .{ }^{b}{ }^{c}$ Ref. $38 .{ }^{c}$ Ref. $40 .{ }^{d}$ Ref. 2. & \\
\hline
\end{tabular}

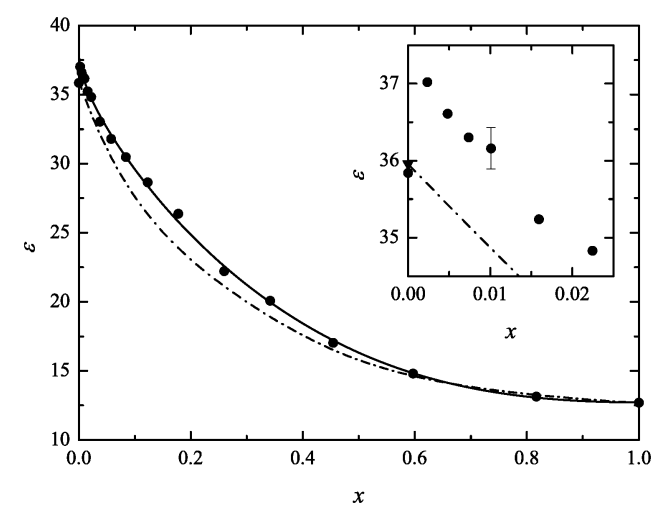

Fig. 3 Static relative permittivity, $\varepsilon(\bullet$, solid line as a guide to the eye; $\boldsymbol{\nabla}$ ref. 40), as a function of IL mole fraction, $x$, of mixtures of $\left[\mathrm{C}_{6} \mathrm{mim}\right]^{+}\left[\mathrm{Tf}_{2} \mathrm{~N}\right]^{-}+$acetonitrile at $25{ }^{\circ} \mathrm{C}$. The dash-dotted line indicates the static permittivity expected from the molar concentrations of acetonitrile and IL present in the mixtures (see the text).

investigated IL concentration, $x=0.00235$ (inset of Fig. 3). A similar behaviour was observed for mixtures of ILs with dichloromethane. ${ }^{3,25}$ Additionally, for $x<0.6$ the static permittivity of the mixtures estimated from the analytical concentrations of IL and acetonitrile (dash-dotted line in Fig. 3) with the help of the effective dipole moments of the pure components ${ }^{3}$ is significantly smaller than the experimental data. These observations are strong indication for the presence of an additional species with large dipole moment at low IL content. Most likely, this is a $\left[\mathrm{C}_{6} \mathrm{mim}\right]^{+}\left[\mathrm{Tf}_{2} \mathrm{~N}\right]^{-}$ion pair. Note that ion pairs of imidazolium ILs were not only found in dichloromethane $\mathrm{e}^{3,25}$ but also in acetonitrile and even water. ${ }^{42}$

\subsection{Solvatochromy of $12^{\prime} \mathrm{CA}$ in the IL/organic solvent mixture}

Fig. 4a shows the dependence of the $\mathrm{S}_{0} \rightarrow \mathrm{S}_{2}$ absorption band of $12^{\prime} \mathrm{CA}$ on mixture composition. For the sake of clarity, only the maximum absorption region of the normalized spectra in the range $0.9-1.0$ is presented. Fig. $4 \mathrm{~b}$ contains a plot of the 

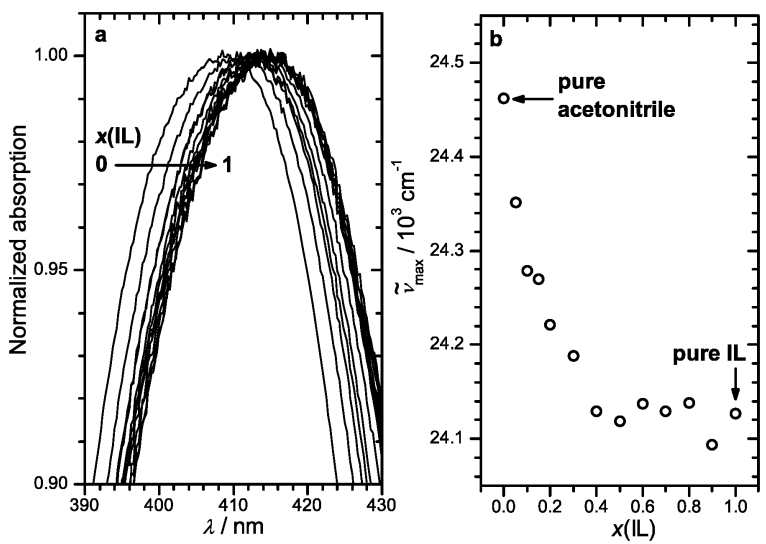

Fig. 4 (a) Maximum region of the steady-state absorption spectra of $12^{\prime} \mathrm{CA}$ in $\left[\mathrm{C}_{6} \mathrm{mim}\right]^{+}\left[\mathrm{Tf}_{2} \mathrm{~N}\right]^{-}$/acetonitrile mixtures as a function of wavelength; (b) absorption maxima (in $\mathrm{cm}^{-1}$ ) as a function of $x(\mathrm{IL})$.

Table 2 Index of refraction $n$, solvent polarizability function $R(n)$, steady-state $\mathrm{S}_{0} \rightarrow \mathrm{S}_{2}$ absorption maximum $\tilde{v}_{\max }$, internal conversion time constant $\tau_{1}\left(\mathrm{~S}_{1} / \mathrm{ICT} \rightarrow \mathrm{S}_{0}\right)$ and fast contribution to solvent relaxation $\tau_{3}$ of $12^{\prime} \mathrm{CA}$ in mixtures of $\left[\mathrm{C}_{6} \mathrm{mim}\right]^{+}\left[\mathrm{Tf}_{2} \mathrm{~N}\right]^{-}$and acetonitrile

\begin{tabular}{llllll}
\hline$x(\mathrm{IL})$ & $n$ & $R(n)^{a}$ & $\tilde{v}_{\max } / \mathrm{cm}^{-1}$ & $\tau_{1} / \mathrm{ps}$ & $\tau_{3} / \mathrm{ps}$ \\
\hline 0.00 & 1.343 & 0.211 & 24462 & $42 \pm 1$ & $0.5 \pm 0.1$ \\
0.05 & 1.365 & 0.224 & 24352 & $38 \pm 2$ & $0.8 \pm 0.1$ \\
0.10 & 1.381 & 0.232 & 24279 & $39 \pm 1$ & $1.1 \pm 0.1$ \\
0.15 & 1.392 & 0.238 & 24269 & $43 \pm 3$ & $1.3 \pm 0.1$ \\
0.20 & 1.399 & 0.242 & 24221 & $45 \pm 3$ & $1.5 \pm 0.2$ \\
0.30 & 1.410 & 0.248 & 24188 & $56 \pm 1$ & $1.8 \pm 0.1$ \\
0.40 & 1.416 & 0.251 & 24130 & $66 \pm 3$ & $2.1 \pm 0.5$ \\
0.50 & 1.420 & 0.253 & 24119 & $72 \pm 2$ & $1.9 \pm 0.3$ \\
0.60 & 1.423 & 0.255 & 24137 & $83 \pm 5$ & $2.5 \pm 0.9$ \\
0.70 & 1.426 & 0.256 & 24130 & - & - \\
0.80 & 1.427 & 0.257 & 24138 & $85 \pm 4$ & $2.3 \pm 0.5$ \\
0.90 & 1.429 & 0.258 & 24093 & $91 \pm 2$ & $2.4 \pm 0.4$ \\
1.00 & 1.430 & 0.258 & 24127 & $94 \pm 4$ & $2.0 \pm 0.1$
\end{tabular}

${ }^{a}$ Solvent polarizability function: $R(n)=\left(n^{2}-1\right) /\left(n^{2}+2\right)$.

corresponding maximum position $\tilde{v}_{\max } v s$. IL mole fraction (for values see Table 2). There is a clear nonlinear red-shift of the steady-state absorption spectrum with increasing $x(\mathrm{IL})$. For the $12^{\prime} \mathrm{CA}$ probe molecule and a range of other carotenoids, we previously demonstrated in organic solvents and supercritical fluids that the absorption shift depends linearly on the polarizability function $R(n)=\left(n^{2}-1\right) /\left(n^{2}+2\right)$, a behavior which is typical for many carotenoids. ${ }^{35,43}$ In the current study, we were able to confirm this behavior for the IL/organic solvent mixture: Fig. 5 contains a plot of the absorption maximum against $R(n)$, with our measured refractive indices $n$ and $R(n)$ values summarized in Table 2 . The linear dependence clearly suggests that the bulk composition of the mixture is a good approximation to the local mixture composition in the vicinity of the probe, i.e., there is no indication for "preferential" solvation of the probe molecule by either of the mixture components. This finding is in line with previous studies on other types of mixtures, such as gas-expanded liquids. ${ }^{28}$ We note that including the absorption maxima of $12^{\prime} \mathrm{CA}$ for the previously studied organic solvents and ILs ${ }^{19,20}$ (not shown in Fig. 5) still leads to the same qualitative dependence on polarizability, however with a stronger scatter

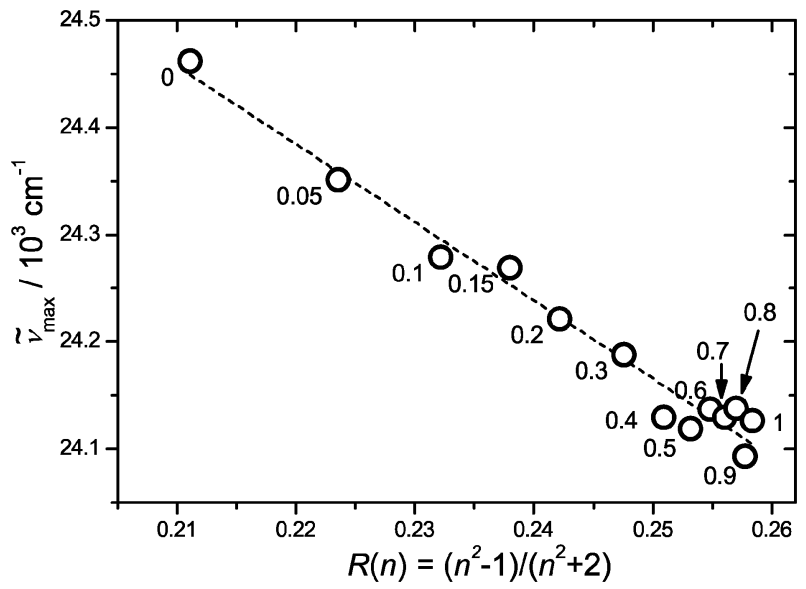

Fig. 5 Steady-state absorption maxima of $12^{\prime} \mathrm{CA}$ as a function of the solvent polarizability function $R(n)$ for different $x(\mathrm{IL})$ (numbers).

of the points. This is understandable considering the strong variation in the solvent type, e.g. regarding $\mathrm{H}$-bonding, shape, size, etc. We also note that results, such as those shown in Fig. $4 b$, have been frequently interpreted as an indication of specific solvation effects. ${ }^{29}$ However, it is clear that the nonlinearities in Fig. $4 \mathrm{~b}$ can be simply traced back to the much larger molar polarizability of the IL compared to acetonitrile.

\section{3 $\mathrm{S}_{1} / \mathrm{ICT}$ lifetime as a function of mixture composition}

For a first overview, Fig. 6 compares selected PSCP broadband absorption spectra for $\left[\mathrm{C}_{6} \mathrm{mim}\right]^{+}\left[\mathrm{Tf}_{2} \mathrm{~N}\right]^{-}$, acetonitrile and four selected mixture compositions at $10 \mathrm{ps}$. All spectra consist of a negative-going $\mathrm{S}_{0} \rightarrow \mathrm{S}_{2}$ ground state bleach (GSB, 350-450 nm) and the characteristic double-peak of the $\mathrm{S}_{1} / \mathrm{ICT} \rightarrow \mathrm{S}_{n}$ excited state absorption (ESA, peaks at ca. 500 and $600 \mathrm{~nm}$ ). We note that an additional smaller ESA band peaking at $c a$. $720 \mathrm{~nm}$ arises from absorption of the $12^{\prime} \mathrm{CA}$ radical cation (presumably the $\mathrm{D}_{0} \rightarrow \mathrm{D}_{3}$ transition). The radical cation is produced by two-photon ionization,

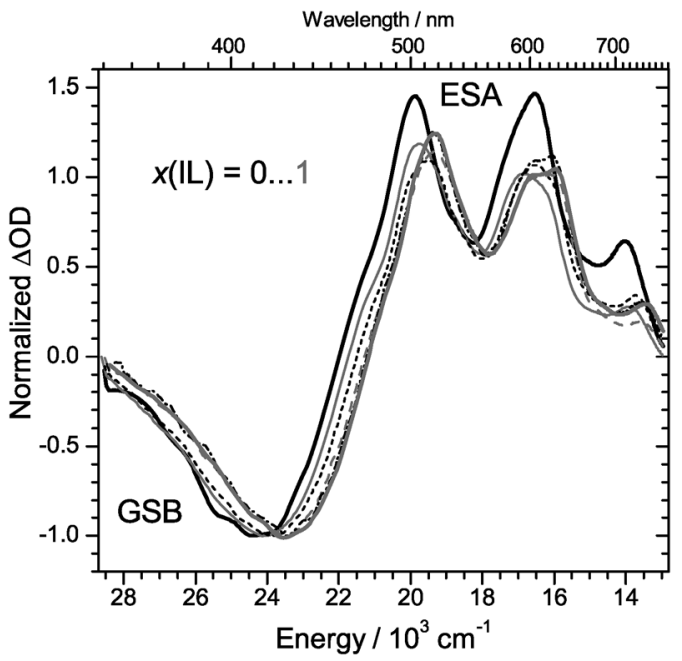

Fig. 6 PSCP spectra for $12^{\prime} \mathrm{CA}$ mixtures of $\left[\mathrm{C}_{6} \mathrm{mim}\right]^{+}\left[\mathrm{Tf}_{2} \mathrm{~N}\right]^{-}$and acetonitrile at $10 \mathrm{ps}$ as a function of $x(\mathrm{IL})$ : (black) $x(\mathrm{IL})=0$; (thin grey) $x(\mathrm{IL})=0.1 ;($ dotted black) $x(\mathrm{IL})=0.25 ;$ (dashed grey) $x(\mathrm{IL})=$ 0.5 ; (dash-dotted) $x(\mathrm{IL})=0.75$; and (thick grey line) $x(\mathrm{IL})=1$. 
when a sufficiently high pump pulse energy is used. ${ }^{22}$ At long times (not shown), after the $\mathrm{S}_{1} / \mathrm{ICT} \rightarrow \mathrm{S}_{n}$ ESA band of neutral $12^{\prime} \mathrm{CA}$ has completely decayed, only the characteristic longlived absorption of the $12^{\prime} \mathrm{CA}$ radical cation absorption remains, with a peak at $720 \mathrm{~nm}$ and an extended tail toward shorter wavelengths (see e.g. Fig. 2 in ref. 22, which also shows the remaining GSB due to the missing $\mathrm{S}_{0}$ population of neutral $12^{\prime} \mathrm{CA}$ ). Under the selected excitation conditions of the current PSCP experiments, this radical cation absorption overwhelms the well-known $\mathrm{S}_{1} / \mathrm{ICT} \rightarrow \mathrm{S}_{0}$ stimulated emission of neutral $12^{\prime} \mathrm{CA}^{20}$ The trends observed for the transient absorption spectra confirm the larger red-shift with increasing mole fraction of IL (Fig. 4) also for the $\mathrm{S}_{1} / \mathrm{ICT} \rightarrow \mathrm{S}_{n}$ ESA band and the cation absorption band.

Next, we recorded transient pump-probe absorption signals of $12^{\prime} \mathrm{CA}$ for different mole fractions of IL at extremely low pump power, which suppresses the formation of the radical cation completely. Two representative examples are shown in Fig. 7 for $x(\mathrm{IL})=0.1$ and 0.9 , respectively $\left(\lambda_{\text {pump }}=430 \mathrm{~nm}\right.$, $\lambda_{\text {probe }}=860 \mathrm{~nm}$ ). The typical signal shape consists of a transient $\mathrm{S}_{2} \rightarrow \mathrm{S}_{n}$ absorption peak at early times which due to the internal conversion (IC) process $\mathrm{S}_{2} \rightarrow \mathrm{S}_{1} /$ ICT quickly develops into pronounced $\mathrm{S}_{1} / \mathrm{ICT} \rightarrow \mathrm{S}_{0}$ stimulated emission (SE), with $\tau_{2}=120 \mathrm{fs}$. The SE finally decays because of the IC process $\mathrm{S}_{1} / \mathrm{ICT} \rightarrow \mathrm{S}_{0}$. This decay directly provides the $\mathrm{S}_{1} / \mathrm{ICT}$ lifetime $\tau_{1}$, which is summarized in Table 2 for all mixtures.

Table 2 clearly demonstrates that there is a pronounced increase of the lifetime upon addition of IL. The fast decay of pure acetonitrile (42 ps) is expected for such a polar solvent and is slightly faster than for methanol (49 ps). The value for pure $\left[\mathrm{C}_{6} \mathrm{mim}\right]^{+}\left[\mathrm{Tf}_{2} \mathrm{~N}\right]^{-}(94 \mathrm{ps})$ is consistent with the value measured recently by us. ${ }^{20}$ For comparison of the transient absorption and dielectric data the following strategy was employed: the decrease of $\tau_{1}$ from $\left[\mathrm{C}_{6} \mathrm{mim}\right]^{+}\left[\mathrm{Tf}_{2} \mathrm{~N}\right]^{-}$to

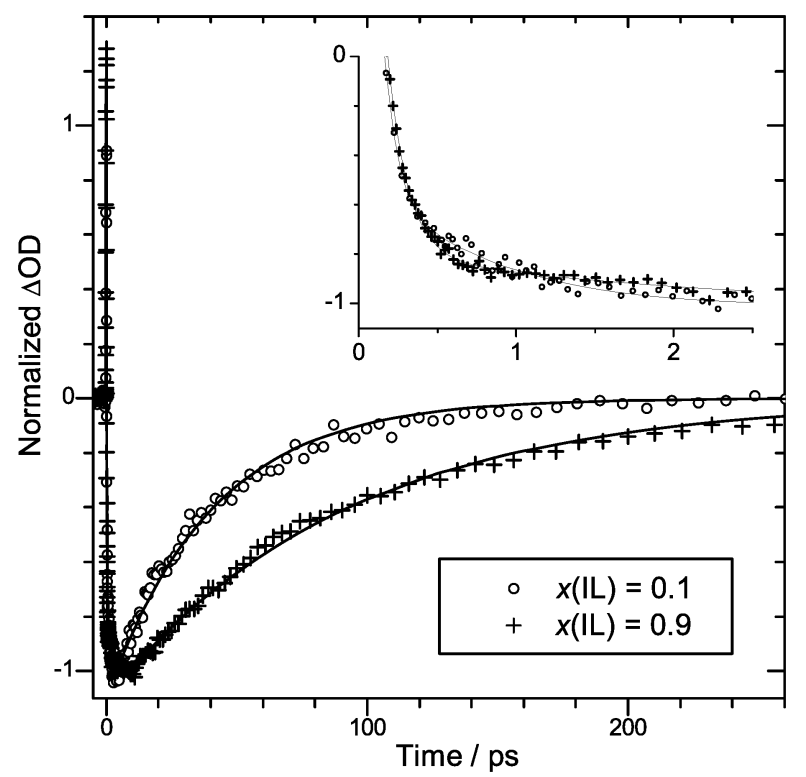

Fig. 7 Time-resolved transient absorption/stimulated emission signals of $12^{\prime} \mathrm{CA}$ in the near IR region for mixtures of $\left[\mathrm{C}_{6} \mathrm{mim}\right]^{+}\left[\mathrm{Tf}_{2} \mathrm{~N}\right]^{-}$ and acetonitrile with $x(\mathrm{IL})=0.1$ and 0.9 . The inset shows a magnification at early times. $\lambda_{\text {pump }}=430 \mathrm{~nm}, \lambda_{\text {probe }}=860 \mathrm{~nm}$. acetonitrile can be understood in terms of a polarity-induced reduction of the $\mathrm{S}_{1} / \mathrm{ICT}-\mathrm{S}_{0}$ energy gap $\Delta E$, which accelerates the nonradiative IC process. ${ }^{16}$ This type of behavior is also well-known for other carbonyl-substituted carotenoids, e.g., peridinin or fucoxanthin. ${ }^{44}$ Such a dependence can be described in terms of an energy-gap-law approach: ${ }^{16,45}$

$$
\ln \left(k_{1} / \mathrm{s}^{-1}\right)=\ln \left(\left\{\tau_{1} / \mathrm{s}\right\}^{-1}\right)=A-B \cdot \Delta E / \mathrm{cm}^{-1}
$$

Here, $k_{1}\left(=\tau_{1}{ }^{-1}\right)$ is the IC rate constant and $A$ and $B$ are fit constants. $\Delta E$ can be approximately related to the Stokes shift $\Delta \tilde{v}_{\text {Stokes }}$, because both quantities are measures of the solventdependent energetic separation between the $\mathrm{S}_{1} / \mathrm{ICT}$ and $\mathrm{S}_{0}$ electronic states. Simple continuum theories of solvation predict that $\Delta \tilde{v}_{\text {Stokes }}$ is proportional to the polarity parameter $\Delta f{ }^{46}$ defined as:

$$
\Delta f=R(\varepsilon)-R(n)=\frac{\varepsilon-1}{\varepsilon+2}-\frac{n^{2}-1}{n^{2}+2} .
$$

Of course, the shortcomings of continuum-type approaches for the description of IL properties are well documented, ${ }^{23,47-49}$ and so far no physically sound model for a reliable description of ILs exists. Still, we believe that one obtains reasonable qualitative trends for the mixtures of the current study by using correlations based on quantities such as $\Delta f$. Therefore, we compare the different experiments by plotting the transient absorption results in terms of $\ln \left(k_{1} / \mathrm{s}^{-1}\right)$ and the dielectric data in terms of $\Delta f v s$. the mole fraction $x(\mathrm{IL})$. This is shown in Fig. 8a, where the $y$ axes are scaled in a way that the $\ln \left(k_{1}\right)$ and $\Delta f$ values for pure acetonitrile and the pure IL are on top of each other to highlight the dependence on mixture

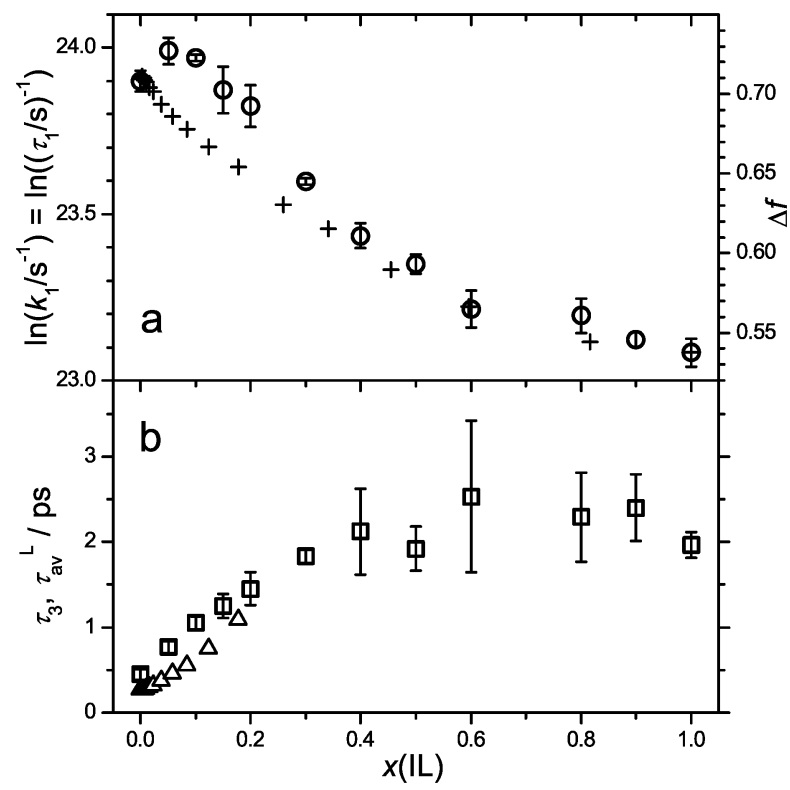

Fig. 8 (a) Open circles with error bars: logarithm of the $\mathrm{S}_{1} / \mathrm{ICT} \rightarrow \mathrm{S}_{0}$ internal conversion rate constant $\ln \left(k_{1} / \mathrm{s}^{-1}\right)=\ln \left(\left(\tau_{1} / \mathrm{s}\right)^{-1}\right)$ as a function of IL mole fraction for $12^{\prime} \mathrm{CA}$ in $\left[\mathrm{C}_{6} \mathrm{mim}\right]^{+}\left[\mathrm{Tf}_{2} \mathrm{~N}\right]^{-}$/acetonitrile mixtures, calculated from the values in Table 2; crosses: $\Delta f$ values calculated using eqn (2) from $\varepsilon$ values in Table 1 and interpolated $n$ values from Table 2. (b) Fast solvation component $\tau_{3}$ of $12^{\prime} \mathrm{CA}$ (open squares with error bars) and average longitudinal relaxation time $\tau_{\mathrm{av}}^{\mathrm{L}}$ from dielectric spectroscopy (open triangles) as a function of $x(\mathrm{IL})$. 
composition. Error bars for the $\ln \left(k_{1}\right)$ values (and also for the $\tau_{3}$ values later on presented in Fig. $8 \mathrm{~b}$ ) were extracted from the fit results of $c a$. 5-15 transients recorded for each $x$ (IL). One observes a similar slightly curved dependence of $\ln \left(k_{1}\right)$ and $\Delta f$ on IL mole fraction. For the data representation chosen, deviations become larger below $x(\mathrm{IL}) \approx 0.4$. Of particular interest is the maximum of $\ln \left(k_{1}\right)$ around $x(\mathrm{IL})=0.05$ meaning that addition of a small amount of IL to pure acetonitrile leads to an acceleration of the IC process. The effect might be due to the formation of discrete ion pairs of the IL with large dipole moment. ${ }^{27}$ As discussed in Section 3.1, a similar behavior is detected by dielectric spectroscopy, which finds a small maximum at $x(\mathrm{IL})=0.00235$ and a larger value of $\varepsilon$ than estimated from the analytical concentrations of IL and acetonitrile for $x(\mathrm{IL})<0.6$ (Fig. 3$){ }^{3}$

We recently demonstrated that the lifetime $\tau_{1}$ of the $\mathrm{S}_{1} / \mathrm{ICT}$ state of $12^{\prime} \mathrm{CA}$ and related apocarotenoids with terminal carbonyl-substitution strongly depends on the dipolarity of the solvent. ${ }^{15-17,19,21}$ Specifically for $12^{\prime} \mathrm{CA}$ in organic solvents, we established a smooth correlation between $\tau_{1}$ and the polarity parameter $\Delta f$. This is shown in Fig. 9. Lifetimes varied between $230 \mathrm{ps}$ in $n$-hexane and $49 \mathrm{ps}$ in methanol. ${ }^{20}$ The current experiment in the highly polar acetonitrile fits very well into this correlation. Interestingly, ILs featuring imidazolium, pyrrolidinium, tetraalkylammonium, and trialkylsulfonium cations did not follow the correlation (open circles in Fig. 9). In all these aprotic ILs, the probe experiences a "local" polarity comparable to short-chain alcohols, ${ }^{20}$ which is higher than one would expect based on the static dielectric constants extrapolated by dielectric spectroscopy. ${ }^{1,2,4,50-53}$ Instead, for a series of chemically and structurally different cations featuring the same counter-anion $\left[\mathrm{Tf}_{2} \mathrm{~N}\right]^{-}$, we succeeded in establishing

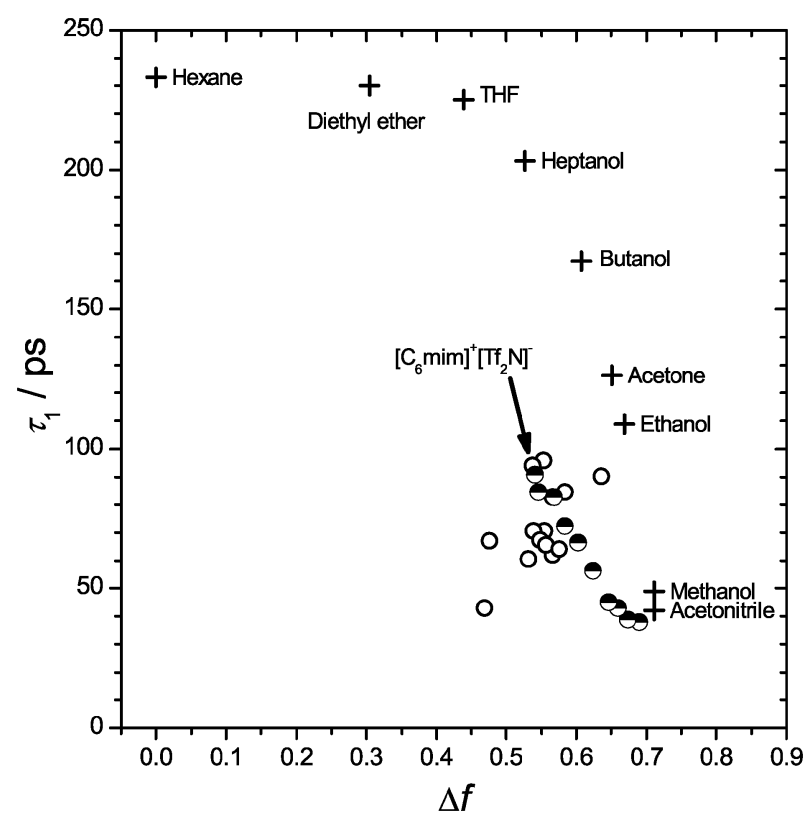

Fig. $9 \mathrm{~S}_{1} / \mathrm{ICT} \rightarrow \mathrm{S}_{0}$ internal conversion time constants $\tau_{1}$ of $12^{\prime} \mathrm{CA}$ as a function of the solvent dipolarity parameter $\Delta f$. Crosses: organic solvents reported in ref. 20 (acetonitrile from this work). Open circles: ILs from ref. 20 with new/revised $\varepsilon$ values from ref. 1 and $2\left(\left[\mathrm{C}_{6} \mathrm{mim}\right]^{+}\left[\mathrm{Tf}_{2} \mathrm{~N}\right]^{-}\right.$ from this work). Half-filled circles: $\left[\mathrm{C}_{6} \mathrm{mim}\right]^{+}\left[\mathrm{Tf}_{2} \mathrm{~N}\right]^{-}$/acetonitrile mixtures from this work. an empirical correlation between $\tau_{1}$ and the IL cation radius, which suggests the importance of Coulombic interactions of cations with the negatively charged carbonyl end of the reporter molecule. ${ }^{20}$ This probably leads to a stronger stabilization of the $\mathrm{S}_{1} /$ ICT state of the probe.

The current work allows us to investigate the applicability of this correlation to IL mixtures with the polar aprotic solvent acetonitrile. For that, we use $\Delta f$ values directly calculated from the static dielectric constants (Table 1) and the refractive indices (Table 2). The results are included in Fig. 9 as half-filled circles. The mixtures smoothly connect the highly polar part of the $\tau_{1}-\Delta f$ correlation with the IL region. As a result, mixtures with increasing IL composition progressively deviate from the correlation. This behavior supports our previous interpretation that simple descriptions based on dielectric continuum theory, which work surprisingly well for dipolar organic solvents, fail for imidazolium-based ILs, because (cation) monopole-dipole contributions dominate the IL cation-probe interaction. In fact, this is also consistent with our previous finding that an addition of a large amount of $\mathrm{Li}^{+}$ cations to organic solvents markedly reduces $\tau_{1}$ of $12^{\prime} \mathrm{CA} .^{20}$

\subsection{Solvation dynamics as observed by the local probe and dielectric spectroscopy}

The transient absorption signals contain additional information related to the solvation response upon the change of the probe's dipole moment. Because the $\mathrm{S}_{1} / \mathrm{ICT}$ lifetime of $12^{\prime} \mathrm{CA}$ in the mixtures studied is fairly short $(<100 \mathrm{ps})$, we restrict our discussion to the short time dynamics. It was recently shown for several systems that ILs exhibit a biphasic solvation response, with an ultrashort and a much slower contribution, where the latter one can be well described by a stretched-exponential function. ${ }^{21-23,54}$ The former one corresponds to high frequency solvent modes in the dielectric spectrum. In the near-IR transient absorption experiments, the ultrafast response is visible at early times as a curvature on the signals which is due to the transient Stokes shift of the SE band of $12^{\prime} \mathrm{CA}$, see the inset in Fig. 7. Fitting of the transients provides an additional time constant $\tau_{3}$, which is plotted as a function of IL mole fraction in Fig. 8b including error bars deduced from typically 5-15 experiments at a given mole fraction. Values are summarized in Table 2. Regarding possible variations of $\tau_{3}$ with probe wavelength, we note that for another closely related carbonyl carotenoid system shifts in the probe wavelength on the order of $20 \mathrm{~nm}$ (upper limit estimated from the spectral shift seen in Fig. 6) should not change $\tau_{3}$ by more than typically $10 \% .^{17}$

For pure acetonitrile, the response is extremely fast, which is in agreement with findings for other probe molecules, such as coumarin $153 .{ }^{23,55}$ For the pure IL, this response is considerably slower, around 2 ps. The interesting observation is that already at low IL mole fractions the solvation response is markedly slowed down. We tentatively explain this by a perturbation of the ultrafast cooperative response of the acetonitrile network by the constituents of the IL and the increasing viscosity of the mixture upon adding IL. Such an interpretation is consistent with a recent Raman spectroscopic study of Aleksa et al. ${ }^{56}$ and earlier results of computer simulations for acetonitrile and $\mathrm{CO}_{2}$ by Ladanyi and Maroncelli. ${ }^{57}$ 
Further support for this view comes from a comparison with the results from dielectric spectroscopy. The connection between solvation dynamics and dielectric relaxation of the solvent is through the longitudinal relaxation time, $\tau^{\mathrm{L}}{ }^{58}$ For a solute dipole embedded in a solvent showing Debye-type relaxation the latter is approximately given by

$$
\tau^{\mathrm{L}}=\tau \frac{3 \varepsilon_{\infty}}{2 \varepsilon+\varepsilon_{\infty}} .
$$

Such a simple relation does not exist for the present samples but may be tentatively used with $\tau_{\mathrm{av}}=\tau$ for $x<0.2$ because of the predominance of the acetonitrile contribution to the spectra (Fig. 2). Quantitative agreement of $\tau_{\mathrm{av}}^{\mathrm{L}}$ with $\tau_{3}$ from the transient absorption signals cannot be expected because of the approximate nature of the average relaxation time, $\tau_{\mathrm{av}}$. However, at low IL content $\tau_{\mathrm{av}}^{\mathrm{L}}$ nicely parallels the increase of $\tau_{3}$ (Fig. 8b) corroborating the interpretation of $\tau_{3}$ as an indicator for fast solvation dynamics. In fact, there might be even a slight systematic shift in $\tau_{3}$ values, because due to the first ultrafast $\mathrm{S}_{2} \rightarrow \mathrm{S}_{1} /$ ICT IC step ( $c a .120 \mathrm{fs}$ ) the fastest part of the solvation response might be somewhat "smeared out". This would mean that the $\tau_{3}$ values from transient absorption should be taken as upper limits. This effect would be more pronounced for the acetonitrile-rich mixtures showing fast relaxation. In fact, such a reduction, possibly by perhaps $0.1-0.2 \mathrm{ps,} \mathrm{would} \mathrm{actually}$ bring $\tau_{3}$ even closer to $\tau_{\mathrm{av}}^{\mathrm{L}}$ from DRS (Fig. 8b).

\section{Conclusions}

The combination of dielectric relaxation spectroscopy (DRS) and ultrafast transient absorption techniques has provided a comprehensive view of the polarity and dynamics of $\left[\mathrm{C}_{6} \mathrm{mim}\right]^{+}\left[\mathrm{Tf}_{2} \mathrm{~N}\right]^{-}+$acetonitrile mixtures. DRS finds a strong decrease of the static dielectric constant from acetonitrile (ca. 35.9) to the pure IL (12.7). For vanishing IL content, however, there is no smooth extrapolation of $\varepsilon$ to the value of pure acetonitrile, and instead the value $\varepsilon=37.0$ is found at the lowest IL content investigated $(x=0.00235)$. This effect can be most likely traced back to the influence of $\left[\mathrm{C}_{6} \mathrm{mim}\right]^{+}\left[\mathrm{Tf}_{2} \mathrm{~N}\right]^{-}$ion pairs. The picture is confirmed by ultrafast transient absorption measurements employing the local polarity and $\mathrm{THz}$ probe $12^{\prime}$ apo- $\beta$-carotenoic- $12^{\prime}$-acid $\left(12^{\prime} \mathrm{CA}\right)$. The lifetime $\tau_{1}$ of the probe's $\mathrm{S}_{1} /$ ICT state, which is a convenient indicator of polarity, shows a strong increase from pure acetonitrile to the pure IL, corresponding to a substantial decrease of polarity with increasing IL content in the mixture. Interestingly, as in DRS, at small IL concentrations the local environment of the probe appears to be slightly more polar than that in pure acetonitrile which again points toward an influence of IL ion pairs at low IL content. In any case, the linear shift of the $\mathrm{S}_{0} \rightarrow \mathrm{S}_{2}$ absorption maximum with mixture polarizability strongly indicates that "preferential solvation" of the probe is negligible.

Using the static dielectric constants from DRS, one finds that the probe's lifetime does not follow the correlation with the dipolarity parameter $\Delta f$, which was previously established in organic solvents. ${ }^{20}$ The behavior, which becomes more and more evident with increasing IL content, points toward a substantial stabilization of the $12^{\prime} \mathrm{CA} \mathrm{S}_{1} / \mathrm{ICT}$ state by charge-dipole interactions of IL cation(s) with the terminal carbonyl group.
The $12^{\prime} \mathrm{CA}$ probe also identified a fast solvent relaxation component which is around 0.5 ps in acetonitrile and about $2 \mathrm{ps}$ in the pure IL. A small addition of IL to acetonitrile is already sufficient to slow down the reorientation dynamics of the acetonitrile network and/or increase the viscosity of the mixture. The picture is in agreement with the results from DRS which finds an increase of the average longitudinal relaxation time in the same range of mixture composition.

For future studies, it will be worthwhile to extend this combined approach to other IL mixtures, containing, e.g., more polar ILs or less polar organic solvents, in order to obtain a more complete overview of the dynamics in these interesting binary solvent systems.

\section{Acknowledgements}

We are thankful to H. Ernst and B. Schäfer (BASF SE) for providing the $12^{\prime}$-apo- $\beta$-carotenoic- $12^{\prime}$-acid samples and discussions about carotenoids. We also thank N.P. Ernsting and J.L. Pérez Lustres for help during the implementation of the PSCP setup, J. Troe and A.M. Wodtke for on-going support, as well as R. Bürsing for excellent technical assistance during the experiments. The Regensburg and Siegen projects have been funded by the DFG priority programme SPP 1191 "Ionic Liquids". We also thank the referees for their valuable comments.

\section{Notes and references}

1 M.-M. Huang, Y. Jiang, P. Sasisanker, G. W. Driver and H. Weingärtner, J. Chem. Eng. Data, 2011, 56, 1494.

2 J. Hunger, A. Stoppa, S. Schrödle, G. Hefter and R. Buchner, ChemPhysChem, 2009, 10, 723.

3 J. Hunger, A. Stoppa, R. Buchner and G. Hefter, J. Phys. Chem. B, 2009, 113, 9527.

4 C. Wakai, A. Oleinikova, M. Ott and H. Weingärtner, J. Phys. Chem. B, 2005, 109, 17028.

5 D. A. Turton, J. Hunger, A. Stoppa, G. Hefter, A. Thoman, M. Walther, R. Buchner and K. Wynne, J. Am. Chem. Soc., 2009, 131, 11140.

6 M. Krüger, E. Bründermann, S. Funkner, H. Weingärtner and M. Havenith, J. Chem. Phys., 2010, 132, 101101.

7 D. A. Turton, T. Sonnleitner, A. Ortner, M. Walther, G. Hefter, K. R. Seddon, S. Stana, N. V. Plechkova, R. Buchner and K. Wynne, Faraday Discuss., 2012, 154, 145.

8 C. Schröder, T. Sonnleitner, R. Buchner and O. Steinhauser, Phys. Chem. Chem. Phys., 2011, 13, 12240.

9 C. Schröder, J. Hunger, A. Stoppa, R. Buchner and O. Steinhauser, J. Chem. Phys., 2008, 129, 184501.

10 C. Schröder, C. Wakai, H. Weingärtner and O. Steinhauser, J. Chem. Phys., 2007, 126, 084511.

11 S. N. V. K. Aki, J. F. Brennecke and A. Samanta, Chem. Commun., 2001, 413.

12 C. Reichardt, Green Chem., 2005, 7, 339

13 A. Oehlke, K. Hofmann and S. Spange, New J. Chem., 2006, 30, 533 .

14 R. Lungwitz, V. Strehmel and S. Spange, New J. Chem., 2010, 34, 1135.

15 D. A. Wild, K. Winkler, S. Stalke, K. Oum and T. Lenzer, Phys. Chem. Chem. Phys., 2006, 8, 2499.

16 F. Ehlers, D. A. Wild, T. Lenzer and K. Oum, J. Phys. Chem. A, 2007, 111, 2257.

17 M. Kopczynski, F. Ehlers, T. Lenzer and K. Oum, J. Phys. Chem. A, 2007, 111, 5370 .

18 F. Ehlers, T. Lenzer and K. Oum, J. Phys. Chem. B, 2008, 112, 16690.

19 S. Stalke, D. A. Wild, T. Lenzer, M. Kopczynski, P. W. Lohse and K. Oum, Phys. Chem. Chem. Phys., 2008, 10, 2180. 
20 P. W. Lohse, R. Bürsing, T. Lenzer and K. Oum, J. Phys. Chem. B, 2008, 112, 3048.

21 K. Oum, P. W. Lohse, F. Ehlers, M. Scholz, M. Kopczynski and T. Lenzer, Angew. Chem., Int. Ed., 2010, 49, 2230.

22 P. W. Lohse, F. Ehlers, K. Oum, M. Scholz and T. Lenzer, Chem. Phys., 2010, 373, 45.

23 M. Maroncelli, X.-X. Zhang, M. Liang, D. Roy and N. P. Ernsting, Faraday Discuss., 2012, 154, 409.

24 A. Dallmann, M. Pfaffe, C. Mügge, R. Mahrwald, S. A. Kovalenko and N. P. Ernsting, J. Phys. Chem. B, 2009, 113, 15619.

25 J. Hunger, A. Stoppa, R. Buchner and G. Hefter, J. Phys. Chem. B, 2008, 112, 12913.

26 A. Stoppa, R. Buchner and G. Hefter, J. Mol. Liq., 2010, 153, 46.

27 G. Hefter, R. Buchner, J. Hunger and A. Stoppa, in Ionic Liquids: From Knowledge to Application, ed. N. V. Plechkova, R. D. Rogers and K. R. Seddon, American Chemical Society, Washington, DC, 2010, vol. 1030.

28 H. Li, S. Arzhantsev and M. Maroncelli, J. Phys. Chem. B, 2007, 111, 3208.

29 J. L. Gohres, C. L. Kitchens, J. P. Hallett, A. V. Popov, R. Hernandez, C. L. Liotta and C. A. Eckert, J. Phys. Chem. B, 2008, 112, 4666.

30 R. Buchner, G. T. Hefter and P. M. May, J. Phys. Chem. A, 1999, $103,1$.

31 J. Barthel, R. Buchner, P.-N. Eberspächer, M. Münsterer, J. Stauber and B. Wurm, J. Mol. Liq., 1998, 78, 83.

32 A. Stoppa, J. Hunger and R. Buchner, J. Chem. Eng. Data, 2009, 54, 472.

33 T. Lenzer, S. Schubert, F. Ehlers, P. W. Lohse, M. Scholz and K. Oum, Arch. Biochem. Biophys., 2009, 483, 213.

34 P. W. Lohse, J. Kuhnt, S. I. Druzhinin, M. Scholz, M. Ekimova, T. Oekermann, T. Lenzer and K. Oum, Phys. Chem. Chem. Phys., 2011, 13, 19632.

35 K. Golibrzuch, F. Ehlers, M. Scholz, R. Oswald, T. Lenzer, K. Oum, H. Kim and S. Koo, Phys. Chem. Chem. Phys., 2011, 13, 6340 .

36 A. L. Dobryakov, S. A. Kovalenko, A. Weigel, J. L. Pérez Lustres, J. Lange, A. Müller and N. P. Ernsting, Rev. Sci. Instrum., 2010, 81, 113106.
37 M. Kopczynski, T. Lenzer, K. Oum, J. Seehusen, M. T. Seidel and V. G. Ushakov, Phys. Chem. Chem. Phys., 2005, 7, 2793.

38 P. Eberspächer, E. Wismeth, R. Buchner and J. Barthel, J. Mol. Liq., 2006, 129, 3.

39 R. Buchner and G. Hefter, Phys. Chem. Chem. Phys., 2009, 11, 8984

40 J. Barthel, R. Neueder and P. Schröder, Electrolyte Data Collection, Part Ic: Conductivities, Transference Numbers, and Limiting Ionic Conductivities of Solutions of Aprotic, Protophobic Solvents I: Nitriles, Dechema, Frankfurt am Main, Germany, 1996.

41 A. Stoppa, Diploma thesis, University of Regensburg, 2009.

42 M. Bešter-Rogac, A. Stoppa, J. Hunger, G. Hefter and R. Buchner, Phys. Chem. Chem. Phys., 2011, 13, 17588.

43 Z. Chen, C. Lee, T. Lenzer and K. Oum, J. Phys. Chem. A, 2006, 110, 11291.

44 H. A. Frank, J. A. Bautista, J. Josue, Z. Pendon, R. G. Hiller, F. P. Sharples, D. Gosztola and M. R. Wasielewski, J. Phys. Chem. $B, 2000,104,4569$.

45 V. Chynwat and H. A. Frank, Chem. Phys., 1995, 194, 237.

46 L. Reynolds, J. A. Gardecki, S. J. V. Frankland, M. L. Horng and M. Maroncelli, J. Phys. Chem., 1996, 100, 10337.

47 H. Jin, G. A. Baker, S. Arzhantsev, J. Dong and M. Maroncelli, J. Phys. Chem. B, 2007, 111, 7291.

48 X. Song, J. Chem. Phys., 2009, 131, 044503.

49 M. N. Kobrak and H. Li, Phys. Chem. Chem. Phys., 2010, 12, 1922

50 A. Stoppa, J. Hunger, R. Buchner, G. Hefter, A. Thoman and H. Helm, J. Phys. Chem. B, 2008, 112, 4854.

51 H. Weingärtner, Z. Phys. Chem., 2006, 220, 1395.

52 S. Schrödle, G. Annat, D. R. MacFarlane, M. Forsyth, R. Buchner and G. Hefter, Chem. Commun., 2006, 1748.

53 H. Weingärtner, Angew. Chem., Int. Ed., 2008, 47, 654.

54 S. Arzhantsev, J. Hui, G. A. Baker and M. Maroncelli, J. Phys. Chem. B, 2007, 111, 4978.

55 M. L. Horng, J. A. Gardecki, A. Papazyan and M. Maroncelli, J. Phys. Chem., 1995, 99, 17311.

56 V. Aleksa, V. Barkauskas, V. Pogorelov, H. Fuess and V. Balevičius, Lith. J. Phys., 2007, 47, 435.

57 B. M. Ladanyi and M. Maroncelli, J. Chem. Phys., 1998, 109, 3204.

58 B. Bagchi, Annu. Rev. Phys. Chem., 1989, 40, 115. 